\title{
ILCEA
}

Revue de l'Institut des langues et cultures

d'Europe, Amérique, Afrique, Asie et Australie

19 | 2014

Acceptabilité et transgression en langues et cultures de spécialité

\section{« Small is beautiful », une idée transgressive dans la communication externe des banques}

"Small is Beautiful", a Transgressive Idea in the External Communication of Banks

Camille Biros

\section{OpenEdition}

Journals

Édition électronique

URL : http://journals.openedition.org/ilcea/2491

DOI : 10.4000/ilcea.2491

ISSN : 2101-0609

Éditeur

UGA Éditions/Université Grenoble Alpes

Édition imprimée

ISBN : 978-2-84310-280-6

ISSN : $1639-6073$

\section{Référence électronique}

Camille Biros, " «Small is beautiful », une idée transgressive dans la communication externe des

banques », ILCEA [En ligne], 19 | 2014, mis en ligne le 27 juin 2014, consulté le 01 mai 2019. URL

http://journals.openedition.org/ilcea/2491 ; DOI : 10.4000/ilcea.2491

Ce document a été généré automatiquement le 1 mai 2019.

(C) ILCEA 


\title{
«Small is beautiful », une idée transgressive dans la communication externe des banques
}

\author{
"Small is Beautiful", a Transgressive Idea in the External Communication of \\ Banks
}

\section{Camille Biros}

1 Il existe une idée répandue concernant le discours écologiste et le discours économique : l'idée qu'ils s'opposent terme à terme. On a longtemps considéré que les valeurs à l'œuvre dans le discours écologiste étaient en contradiction avec les impératifs économiques. Pour certains chercheurs en sciences du langage, on peut même concevoir le discours écologiste comme s'étant construit à partir du discours économique, par un effet de miroir inversé : « Les discours environnementaux seront considérés comme une réaction, une réponse, un refus, une interrogation, une tentative d'élucidation d'un problème, ou une simple reformulation d'une question par rapport à d'autres disponibles, et principalement parmi ceux-ci, les discours économiques. » (Jalencques-Vigouroux, 2006, p. 78) Il est certain qu'on peut mettre à jour de nombreuses contradictions entre les valeurs défendues dans ces deux types de discours. Il faut pourtant relativiser cette opposition qui pourrait reposer sur une conception stéréotypée de ce que sont le discours économique et le discours écologiste. Le développement durable se conçoit dès le départ comme une tentative de dépassement de cette opposition et les exemples de discours économique incluant des préoccupations écologistes ou de discours écologiste présentant des impératifs économiques sont nombreux.

2 Cependant il est une idée, centrale pour le discours écologiste, autour de laquelle se cristallise peut-être cette opposition, non pas avec le discours économique en général, mais avec un certain type de discours économique répandu, celui de l'économie de marché. Il s'agit des préconisations très différentes concernant la croissance et la 
consommation. L'économie de marché repose sur l'idée qu'il faut favoriser la consommation, qui est source de croissance. La croissance est considérée comme un critère de santé pour un système économique. Lorsque c'est la santé du système écologique qui est mise en avant comme la priorité, ces deux valeurs de consommation et de croissance sont fondamentalement remises en question. Les contraintes environnementales imposent des limites à une consommation effrénée et un questionnement de l'impératif de croissance. La compréhension de ces contraintes permet de mettre à jour le fait qu'un système qui ne cherche pas à réduire la consommation ne peut être soutenable à long terme, ce qui pourrait être au cœur des doutes qui entourent la notion de développement durable. Aujourd'hui, les multinationales, parmi lesquelles les banques, sont de plus en plus encouragées à adopter des principes environnementaux. Au Royaume-Uni, des contraintes légales signifient qu'elles se doivent de communiquer sur l'environnement et sur ce qu'elles font pour limiter sa dégradation. Pourtant, l'activité principale d'une banque ne peut être pratiquée si l'on ne cherche pas à favoriser la consommation. Ne faut-il pas penser alors que le discours de ce type d'organisation doit être hermétique à toute idée de limites imposées à la consommation et à la croissance? Il est possible de s'interroger sur les degrés d'acceptabilité de ce principe écologiste dans le discours externe de communication de la banque. Si proposer des freins à la consommation constitue une transgression, on peut s'interroger sur les stratégies de contournement qui permettent néanmoins à la banque de diffuser un discours de respect des ressources naturelles. Nous commencerons par montrer la place centrale occupée par l'idée qu'il est possible de faire plus avec moins en termes de ressources, dans nombreux textes fondateurs du mouvement environnemental. Puis, nous procèderons à une analyse des textes de communication externe de la banque HSBC, banque britannique qui a la plus grande capitalisation boursière d'après le London Stock Exchange. Nous considèrerons les documents de communication externe publiés au Royaume-Uni, en particulier les rapports sur la responsabilité sociale qui sont consultables en ligne par toute personne ou organisation, qu'elle ait des intérêts liés à la banque ou non, qui souhaite se renseigner sur les activités principales et les revenus de celle-ci. La période étudiée pour la présente étude s'étend de 2000 à 2012, car c'est une période cruciale pour le développement de la communication environnementale. En effet, si en 2000 seules quelques entreprises pionnières publient des rapports sur la responsabilité sociale chaque année, dès 2003, toutes les grandes entreprises cotées au London Stock Exchange le font. À partir de 2012, il devient plus courant pour les entreprises de ne publier cette information que sur leur site internet ou dans une section réduite du rapport annuel financier. Il s'agit de comprendre comment l'idée qui nous intéresse est abordée dans cette communication et au nom de quoi elle devient progressivement acceptable. Finalement, il nous faut constater qu'il s'agit encore d'une idée transgressive au vu des stratégies langagières d'évitement qui sont à l'œuvre dans les textes. 


\section{La beauté du petit dans les textes fondateurs du mouvement environnemental}

\subsection{La place de l'argument économique dans les textes fondateurs du mouvement environnemental}

3 Il faut commencer par élucider ce que nous entendons par la beauté du petit, concept élaboré par Schumacher dans son ouvrage intitulé Small is Beautiful (1972). L'idée exprimée grâce à cette expression peut se manifester de différentes façons. Il s'agit tout d'abord de signifier qu'avec moins de moyens matériels, il est possible pour l'humanité d'atteindre un état qualitativement plus élevé. Deuxièmement, l'expression suggère que la croissance en soi ne devrait pas être un critère pour juger de la santé du système économique. Enfin, elle signifie que certains problèmes humains sont mieux gérés à l'échelle locale et que les petites organisations, qu'elles soient à but lucratif, politique, éducatif, ou autre, sont souvent plus efficaces que celles de grande taille pour régler les problèmes humains. Le terme de «beautiful » permet à Schumacher de mettre en avant l'opposition qu'il fait entre la réussite en termes quantitatifs et en termes qualitatifs ainsi que la plus grande priorité qu'il donne au qualitatif.

4 Les idées développées par Schumacher en 1972 ne sont pas neuves pour le mouvement écologiste et les penseurs qui l'ont favorisé. Différentes méthodes permettent d'identifier les textes fondateurs de ce mouvement. La consultation de dictionnaires spécialisés et de manuels sur l'histoire du mouvement environnemental ainsi que l'identification des auteurs les plus cités par les acteurs du domaine environnemental permettent de dresser une liste non exhaustive d'ouvrages qui ont été déterminants pour la construction du mouvement (Biros, 2011, p. 18) : Nature (1849) de R. W. Emerson, Walden (1854) de H. D. Thoreau, Man and Nature (1867) de J. P. Marsh, Breaking New Ground (1947) de G. Pinchot, A Sand and County Almanac (1949) de A. Leopold, Silent Spring (1962) de R. Carson, The Limits to Growth (1972), du Club de Rome, Small is Beautiful (1972) de E. F. Schumacher. De façon très sommaire, il est possible de résumer le contenu de ces ouvrages: ils ont tous en commun de dénoncer la dégradation des ressources naturelles et d'identifier l'industrialisation massive et la société de consommation comme les causes principales de cette dégradation. Parmi ces textes, nous nous concentrerons sur ceux qui adoptent explicitement une perspective économique.

L'auteur du texte que nous présenterons tout d'abord, Thoreau, est un philosophe américain, élève d'Emerson. Dans son ouvrage Walden, il relate une expérience de plusieurs années de vie dans les bois, en autarcie par rapport au système économique. Le premier chapitre de cet ouvrage est intitulé "Economy" et présente la dimension économique de son expérience ainsi que les réflexions que cette situation particulière lui permettent de développer sur la situation économique en général. Le second ouvrage considéré fut écrit par Schumacher. Il s'agit de Small is Beautiful qui se réfère explicitement à l'économie comme indiqué par son sous-titre Economy as if people mattered. Nous allons présenter le contenu de ces deux ouvrages et les perspectives économiques développées par les auteurs. Cette présentation est faite dans l'ordre chronologique, les travaux de Thoreau précédant d'un siècle ceux de Schumacher. La dégradation de l'environnement est présentée, ainsi que dans les autres ouvrages, comme regrettable en soi, d'un point de vue esthétique et du point de vue de la qualité de vie. Mais ce qui est 
particulier à ces deux ouvrages est qu'ils adoptent des arguments économiques pour critiquer le système en place. Les auteurs visent à proposer un système qu'ils jugent plus réaliste d'un point de vue économique puisqu'il permet de mieux prendre en compte des contraintes environnementales bien réelles.

\subsection{Walden ou l'adoption de la simplicité volontaire}

6 Walden est une narration de l'expérience de deux ans de son auteur, qui mène une vie isolée dans une cabane construite dans les bois près de Walden Pond aux États-Unis. Thoreau y donne des détails pratiques sur sa vie quotidienne avec notamment les méthodes utilisées pour construire son habitat et subvenir à ses besoins alimentaires. Les avantages qu'il trouve à cette autonomie acquise par rapport à la société industrielle sont exposés longuement. Son bonheur à vivre en harmonie avec le rythme de la nature, son observation méditative du monde vivant qui l'entoure, sont expliqués. Ses activités du quotidien acquièrent une dimension spirituelle du fait de son immersion dans la nature. Cependant, certains passages font apparaître le caractère très pragmatique de leur auteur et ses considérations d'ordre purement économique ne sont pas laissées de côté. Thoreau dénonce l'absurdité qu'il y a à passer sa vie à travailler dans des conditions très difficiles, comme il peut l'observer chez ses contemporains des classes ouvrières, afin de payer un loyer pour un logement souvent insalubre. Selon l'auteur, il serait légitime pour ces personnes de s'abstraire du marché du travail et du marché de la consommation, comme il le fait lui-même, afin de se loger et de se nourrir à partir de ressources naturelles qui sont disponibles librement pour chacun. Il dénonce les conventions sociales qui font que des biens matériels sont considérés comme essentiels alors qu'on pourrait légitimement s'en passer: "Some things are really necessaries of life in some circles, the most helpless and diseased, which in others are luxuries merely, and in others still are entirely unknown." (Thoreau, 1854, p. 9) La plupart des biens matériels présentés comme des nécessités par ses contemporains sont considérés avec méfiance par l'auteur : "Most of the luxuries, and many of the so-called comforts of life, are not only not indispensable, but positive hinderances to the elevation of mankind. With respect to luxuries and comforts, the wisest have ever lived a more simple and meager life than the poor. » (Thoreau, 1854, p. 14) Il s'agit notamment de tous les objets qui les éloignent d'un état d'harmonie avec la nature. On peut aussi citer les habits, les diktats de la mode obligeant même les personnes les plus pauvres à changer régulièrement le contenu de leur garde-robe et à les payer un prix qui ne correspond pas à leur valeur réelle, par crainte de se faire juger par leurs contemporains s'ils ne les respectent pas.

7 Thoreau démontre qu'il est trompeur de calculer la valeur d'un objet ou d'un service en termes purement monétaires, car cela ne renseigne pas sur les ressources en vie qui ont été nécessaires pour le produire, qu'il s'agisse de la vie humaine et du temps de labeur, ou de la vie animale et végétale nécessaire pour l'obtention des matières premières utilisées. Il remet en question l'efficacité de la société industrielle dans laquelle il vit. Si l'on prend en compte le travail humain et les ressources naturelles utilisées pour obtenir les biens produits, le coût lui semble bien élevé.

If it is asserted that civilization is a real advance in the condition of man-and I think that it is, though only the wise improve their advantage-it must be shown that it has produced better dwellings without making them more costly; and the cost of a thing is the amount of what I will call life which is required to be exchanged for it, immediately or in the long run.

(Thoreau, 1854, p. 11) 
Afin de s'abstraire du cercle vicieux qui fait que les hommes deviennent esclaves de biens matériels dont ils n'ont pas besoin, il préconise plus d'autonomie pour chaque individu dans la conception et la culture des objets et ressources dont il a besoin. La division du travail est un mécanisme qui rend chacun esclave de la société industrielle dans laquelle il vit: "Where is the division of labor to end? And what object does it finally serve? No doubt another may also think for me; but it is not therefore desirable that he should do so to the exclusion of my thinking for myself. » (Thoreau, 1854, p. 46). Une description détaillée de la nourriture qu'il consomme et de la façon dont il se la procure lui permet de conclure que, de ce point de vue, il est facile d'être autonome à petit coût. Du point de vue de son logement et des meubles, les difficultés pour se les procurer ou les fabriquer sont vite surmontées. Il évoque enfin la satisfaction qui lui est donnée par ces activités variées qui lui permettent de vivre : «In short, I am convinced, both by faith and experience, that to maintain one's self on this earth is not a hardship but a pastime, if we will live simply and wisely; as the pursuits of the simpler nations are still the sports of the more artificial. » (Thoreau, 1854, p. 71) Bien sûr, pour cela il est nécessaire de se contenter de moyens matériels limités. Mais loin de constituer un frein à son épanouissement personnel, ces moyens limités en sont le moteur. Ils offrent à l'auteur un esprit libre grâce auquel il peut se concentrer sur ce qu'il considère comme le principal, l'étude de la philosophie et l'observation de la nature, seules vraies sources de bonheur pour l'homme. Il s'agit donc d'une austérité matérielle volontaire, qui permet à l'homme d'être autonome par rapport à un système industriel dont il se trouve sans cela esclave et qui a également pour corollaire négatif la dégradation des ressources naturelles.

\subsection{Small is Beautiful ou la critique de l'impératif de croissance}

Bien que Thoreau fasse découler de l'observation de son cas particulier quelques idées générales sur le système économique, il aborde essentiellement le problème de la consommation du point de vue de l'individu. Small is Beautiful (1972), en revanche, offre une perspective macroéconomique. À la différence de Walden, l'ouvrage est écrit par un économiste. La première idée développée dans cet ouvrage, idée qui se trouvait également dans l'ouvrage du philosophe, est que le fait de ne pas prendre en compte le coût des ressources naturelles utilisées pour obtenir un produit ou service mène à faire des calculs erronés. Le fait que le coût d'un objet ou service ne soit évalué qu'en fonction de sa valeur sur le marché lui apparaît comme une aberration en termes économiques :

The illusion of unlimited powers, nourished by astonishing scientific and technological achievements, has produced the concurrent illusion of having solved the problem of production. The latter illusion is based on the failure to distinguish between income and capital where this distinction matters most. Every economist and businessman is familiar with the distinction and applies it conscientiously and with considerable subtlety to all economic affairs-except where it really matters: namely, the irreplaceable capital which man has not made, but simply found, and without which he can do nothing. (Schumacher, 1972)

Lorsque l'on prend en compte le critère du capital vie, c'est-à-dire les ressources naturelles, animales et humaines utilisées, critère habituellement laissé de côté par les économistes, l'on s'aperçoit que l'industrie moderne est d'une redoutable inefficacité. Le labeur humain et les ressources naturelles nécessaires pour la faire fonctionner ne peuvent être compensés par la relative qualité de ce qu'elle produit. Elle a pour corollaire le développement des inégalités, la réduction du temps de loisir et de développement 
spirituel des hommes et la dégradation des ressources naturelles. Ses résultats, bien que quantitativement importants, ne sauraient compenser ces inconvénients dont elle est la source: "The most striking thing about modern industry is that it requires so much and accomplishes so little. Modern industry seems to be inefficient to a degree that surpasses one's ordinary power of imagination. » (Schumacher, 1972)

11 L'esclavage de l'homme moderne, issu de l'impératif de croissance matérielle, est également dénoncé par Schumacher. Il montre qu'on ne se pose pas la question de la valeur réelle des technologies plus performantes qui sont développées. On les juge simplement du point de vue matériel et pas du tout du point de vue de l'amélioration de la qualité de vie qu'elles supposent. Si l'on s'interrogeait sur les résultats en termes qualitatifs, on conclurait probablement à leur inefficacité :

Modern technology has deprived man of the kind of work that he enjoys most, creative, useful work with hands and brains, and given him plenty of work of a fragmented kind, most of which he does not enjoy at all. It has multiplied the number of people who are exceedingly busy doing kinds of work which, if it is productive at all, is so only in an indirect or 'roundabout' way, and much of which would not be necessary at all if technology were rather less modern. (Schumacher, 1972)

Afin d'éviter cet asservissement à la technologie, il faudrait préférer un mode de vie relativement austère en termes matériels, ce qui permettrait également de diminuer les sources de conflit entre les hommes: "As physical resources are everywhere limited, people satisfying their needs by means of a modest use of resources are obviously less likely to be at each other's throat than people depending upon a high rate of use. " (Schumacher, 1972) L'auteur défend donc l'idée qu'il y aurait de nombreux avantages à se contenter de moins de moyens matériels. C'est un phénomène qui devrait mener à une moindre croissance économique, ce qui lui semble souhaitable car le critère de la croissance est erroné pour juger de la santé d'un système économique : «If a high-growth economy is needed to fight the battle against pollution which itself appears to be the result of high-growth, what hope is there of ever breaking out of this extraordinary circle? » (Schumacher, 1972)

13 La thèse principale développée par Schumacher est que la simplicité matérielle, la modestie dans la taille, la défiance envers la croissance comme critère de réussite, doivent être mises au centre des programmes de développement humain. L'idée qu'il est possible de faire plus avec moins est extrêmement riche d'applications potentielles. C'est une idée reprise aujourd'hui par les tenants de la décroissance (Latouche, 2004). Mais le terme de "décroissance " a une sonorité transgressive, tout comme celui d'" austérité ", dans le discours médiatique et politique actuel. Pourtant, sans aller jusqu'à mentionner ces termes, l'idée qu'il est possible d'obtenir plus de résultats en ayant une moindre consommation progresse, même au cœur du discours d'organisations dont la fonction ne semble pas faciliter l'adoption. L'étude des rapports sur la responsabilité sociale d'une banque internationale comme l'est $\mathrm{HSBC}$ permet de vérifier ce phénomène.

\section{Les critères pour qu'HSBC adopte l'idée de la valeur du moins}

\subsection{Réduire la consommation pour économiser}

14 Dans le discours de communication externe d'une banque, l'on ne s'attend pas à ce que l'apologie de la simplicité matérielle soit faite. Pour exercer son activité principale, une 
banque doit favoriser la consommation; cette organisation réalise des bénéfices lorsque particuliers et organisations consomment au-delà des ressources financières dont elles disposent. Bien loin de considérer la croissance avec défiance, HSBC présente sa mission comme intimement liée au fait de la favoriser: "Throughout our history we have been where the growth is, connecting customers to opportunities. We enable business to thrive and economies to prosper, helping people to fulfill their hopes and dreams and realise their ambitions. » (HSBC, 2012) La consommation effrénée n'est pas non plus préconisée car elle peut mener à des dépenses non nécessaires. Il est notable que lorsqu'une moindre consommation est présentée comme bénéfique, l'argument écologiste n'est pas celui qui est le plus mobilisé. Le premier argument que l'on retrouve dans les rapports d'HSBC est économique. Comme expliqué par Schumacher, les grandes entreprises sont à la fois des entités productrices et des entités consommatrices. En tant qu'entités productrices, elles sont soumises à un impératif de croissance tandis qu'en tant qu'entités consommatrices, elles doivent réduire leurs coûts et tenter de consommer moins (Schumacher, 1972, chapitre 7). Cet argument économique permet de justifier une réduction de la consommation, mais il ne s'agit que d'une réduction très ponctuelle.

15 Ainsi, dans le rapport sur la responsabilité sociale d'HSBC datant de 2003, les économies faites grâce à une gestion plus efficace de l'utilisation des ressources sont mises en avant : "Although we already look for cost-savings from more efficient use of ressources, we believe we need to go further.» $(2003$, p. 3) Les solutions écologiques adoptées par HSBC sont rarement présentées seulement du point de vue de leur impact environnemental. Si le fait de réduire cet impact est mentionné comme un argument, il est souvent présenté comme secondaire par rapport à l'argument économique. Par exemple, dans l'extrait suivant il n'est mentionné qu'en deuxième : «E-procurement helps reduce costs and our impact on the environment » (HSBC, 2005, p. 24). Le même phénomène est à l'œuvre dans cet extrait du rapport de 2007: «saving approximately US\$30 million per annum in energy costs and subsequent savings in $\mathrm{CO}_{2}$ " ( $\mathrm{HSBC}, 2007$, p. 2). Le paragraphe suivant fait également apparaitre l'argument écologiste dans un deuxième temps. Ce qui est mis en avant comme l'argument principal est l'argument économique, qui semble primer par rapport aux préoccupations écologistes également mentionnées :

Energy-saving studies in our Asia-Pacific head office buildings in Hong Kong have led to initiatives that will save $H K \$ 1.1$ million (US\$140,000) annually. The technology introduced will also reduce the energy demand by 1.07 million kilowatt hours and reduce carbon dioxide emissions by 1,050 tonnes per annum. (HSBC, 2004, p. 24)

Il s'agit d'un thème que l'on retrouve dans tous les rapports $\mathrm{HSBC}$, sous différentes formes, comme en 2008 par exemple : « We invested in new technologies that help cut costs as well as carbon emissions » $(2008$, p. 2) ; puis en 2009 : «Our goal is to deliver enhanced service to our customers at a lower cost and with reduced environmental impact» $(2009$, p. 14); et en 2012: « 36 actions on paper reduction which generated US\$6 million in annual savings and reduced our paper consumption by around 50 million sheets » (2012, p. 12). Différents objets de consommation sont mentionnés dans ces initiatives de réduction de la consommation: énergie, papier, empreinte carbone liée au transport, etc. Ce qui est important est que dans tous les cas, la réduction des coûts est mise en avant comme l'argument essentiel. 


\subsection{Réduire la consommation au nom du développement durable}

17 Le développement durable est un terme très utilisé dans le contexte environnemental et dans le contexte économique à partir de 1987 et de la publication du rapport Brundtland qui l'a popularisé (World Commission on Environment and Development, 1987). Qualifié très justement par A. Krieg Planque $d$ '«opérateur de neutralisation de la conflictualité »(2010), il permet, à première vue, de réconcilier autour de projets communs des acteurs dont les objectifs divergent. La définition du développement durable, telle que donnée dans le rapport Brundtland, est un développement qui permet de subvenir aux besoins des générations présentes sans compromettre ceux des générations futures, à partir des trois piliers que sont l'économie, l'environnement et le social. Ces trois aspects doivent être pris en compte selon les tenants du développement durable. L'avantage de ce concept est qu'il permet de rassembler au-delà des clivages idéologiques puisque peu d'acteurs seraient en désaccord avec l'idée que ces trois piliers ne peuvent être ignorés. Il a également des limites puisque les mêmes acteurs présentent une bien moindre unanimité lorsqu'il s'agit de déterminer en quelle proportion chacun de ces aspects doit être pris en compte pour que ce développement soit vraiment durable.

L'adoption du terme "sustainable development» (développement durable) par HSBC est très visible dans les rapports du début de la période étudiée. On trouve par exemple sept occurrences dans le rapport de l'année $2003^{1}$. Le tableau suivant permet de comparer le nombre d'occurrences de ce terme à celle du nom qui en a dérivé «sustainability». Les données du tableau ont été relevées grâce à l'utilisation du concordancier Antconc.

Figure 1. - Fréquence des termes « sustainable development » et « sustainability » dans les rapports sur la responsabilité sociale d'HSBC $(2003,2012)$.

\begin{tabular}{lcccccccccc}
\hline & 2003 & 2004 & 2005 & 2006 & 2007 & 2008 & 2009 & 2010 & 2011 & 2012 \\
\hline $\begin{array}{l}\text { Nb. d'occurrences } \\
\text { sustainable } \\
\text { development }\end{array}$ & 7 & 3 & 0 & 10 & 5 & 0 & 0 & 0 & 0 & 0 \\
$\begin{array}{l}\text { Nb. d'occurrences } \\
\text { sustainability }\end{array}$ & 9 & 22 & 22 & 44 & 161 & 93 & 99 & 62 & 69 & 64 \\
$\begin{array}{l}\text { Longueur du texte } \\
\text { (nb. de caractères) }\end{array}$ & 145588 & 114602 & 139405 & 140576 & 150715 & 112625 & 100425 & 91573 & 147225 & 145475 \\
Nb. de pages & 48 & 32 & 36 & 40 & 36 & 28 & 24 & 24 & 32 & 36 \\
\hline
\end{tabular}

En 2003, on trouve sept occurrences de "sustainable development », en 2004 trois, en 2005 aucune, en 2006 dix, en 2007 cinq, puis à partir de 2008 le terme disparait tout à fait des rapports. Sa fonction d'« opérateur de neutralisation de la conflictualité » (Krieg Planque, 2010) pourrait alors avoir perdu de l'efficacité et ses limites être devenues plus évidentes après des tentatives de mise en place de projets concrets autour de ces thèmes. Moins bien défini, plus vague quant aux objectifs, le nom qui en a dérivé, « sustainability ", gagne en importance à partir de 2007. Il faut ajouter à cela que, à partir de 2007, le rapport sur la responsabilité sociale, intitulé Corporate Social Responsibility Report jusque-là, devient Sustainability Report.

Comme le montre son utilisation dans l'exemple suivant, l'avantage de ce terme par rapport à celui de développement durable est qu'il permet de maintenir une certaine ambiguité : " $H S B C$ is determined to be one of the world's leading companies in addressing the challenges of embedding sustainability into its business. We believe that doing so will strengthen 
the HSBC brand, helping to deliver long-term value to our stakeholders. » $(2007$, p. 6) Le but est-il la durabilité de l'environnement, la durabilité de l'économie, ou la durabilité de l'entreprise elle-même? Le développement durable invitait à réguler la consommation des ressources, celui de « sustainability » est moins explicite à ce sujet.

La tendance consistant à remplacer le terme de "sustainable development " par celui de " sustainability » n'est cependant pas une particularité du discours externe de la banque. On observe un phénomène comparable dans la communication de nombreuses organisations. Ainsi un corpus de 3550624 termes que nous avons constitué à partir de discours de communication externe de différents types d'organisations situées au Royaume-Uni (Organisations non gouvernementales, entreprises, agences gouvernementales), nous a permis de vérifier la popularité grandissante du terme "sustainability" par rapport à celui de "sustainable development ». Grâce au concordancier Antconc nous avons pu extraire le nombre d'occurrences selon les années, information complétée par le nombre de mots du corpus par année.

Figure 2. - Fréquence des termes « sustainable development » et « sustainability » dans un corpus comparatif.

\begin{tabular}{lccccccccccccc}
\hline & 2000 & 2001 & 2002 & 2003 & 2004 & 2005 & 2006 & 2007 & 2008 & 2009 & 2010 & 2011 & 2012 \\
\hline $\begin{array}{l}\text { sustainable } \\
\text { development }\end{array}$ & 101 & 101 & 132 & 152 & 178 & 191 & 167 & 128 & 70 & 93 & 18 & 3 & 6 \\
$\begin{array}{l}\text { sustainability } \\
\begin{array}{l}\text { Nombre de } \\
\text { mots corpus }\end{array}\end{array}$ & 57048 & 20 & 41 & 235 & 71 & 151 & 85 & 413 & 260 & 591 & 900 & 543 & 129 \\
\hline
\end{tabular}

Il semblerait donc que le discours d'HSBC s'inscrit dans une tendance générale dans son utilisation de ces deux termes. Nous postulons néanmoins que cette tendance générale est significative d'une perte d'intérêt pour le thème de la régulation des ressources. Le terme de "sustainability» permet aux différentes organisations qui l'utilisent d'adopter une posture plus positive et moins contraignante pour ce qui est des programmes environnementaux mis en place.

\subsection{Réduire la consommation en réponse à des contraintes extérieures}

Si le respect de l'environnement en général n'est pas un critère considéré par la banque comme décisif pour justifier une réduction de la consommation, certaines situations, présentées comme des situations de crise, contraignent à prendre des mesures plus respectueuses de l'environnement. Au cours de la période étudiée, c'est-à-dire entre 2000 et 2012, à deux reprises, HSBC se présente comme confrontée à un phénomène inquiétant qui l'oblige à repenser sa propre organisation et notamment à réduire la consommation de certaines ressources. Le premier phénomène de ce type est le changement climatique. La publication du rapport Stern fin 2006, suivie de près de celle du $2^{\mathrm{e}}$ rapport du Groupe d'experts intergouvernemental sur l'évolution du climat (IPCC, 2007) début 2007, ont contribué à la médiatisation inégalée de ce problème environnemental durant l'année 2007. Dans le rapport HSBC datant de 2007, ce contexte est prégnant et les références au changement climatique sont nombreuses. Un indicateur du fait que cette question est jugée prioritaire est la nomination, comme conseiller spécial de la banque, de Lord Stern, spécialiste de l'économie du changement climatique et auteur du rapport publié en 2006: "The appointment of Lord Stern as Special Advisor on Economic Development and Climate Change to the Group Chairman reflects HSBC's view that climate change is the single 
greatest economic, environmental and social challenge we face this century. » $(2007$, p. 13$)$ Le fait qu'il existe un consensus autour de la nécessité de réduire les émissions de $\mathrm{CO}_{2} \mathrm{y}$ est également souligné : "No simple solution to climate change exists, but there is a growing consensus that carbon dioxide emissions must stabilize and then be reduced in order to limit the effects of climate change. » (2007, p. 15) Dans ce contexte, la réduction de la consommation est présentée comme devant être faite, non au nom de principes environnementaux, mais parce que c'est une nécessité dans la mesure où $\mathrm{HSBC}$, comme toute grande organisation, fait face à une situation de crise.

Cependant, une mobilisation de grande ampleur autour de ce sujet n'est pas durable. À partir de 2008 déjà, le caractère prioritaire du changement climatique est atténué. Dans la lettre d'introduction du rapport, le CEO (Chief Executive Officer) explique qu'il y a un recentrage des thématiques du rapport sur les activités principales de la banque, même si le changement climatique n'est pas négligé : « Although this report, in response to stakeholder feedback, focuses more this year on how we are addressing the social impacts of our business activity, environmental sustainability is still very much a focus of our efforts. » $(2008, p$. 2) Cette atténuation devient plus marquée dans les rapports suivants en termes de nombres d'occurrences. Pour l'expliquer, on peut noter qu'à partir de 2010, dans le monde politique au Royaume-Uni, le changement climatique n'est plus jugé comme un thème prioritaire, en particulier par le parti conservateur, chez lequel on voit se développer un certain climato-scepticisme (Carter, 2013). On ne peut plus parler d'un consensus sur ce thème puisque des dissensions d'ordre idéologique concernant son existence sont exprimées. Son caractère factuel et idéologiquement neutre est remis en question et $\mathrm{HSBC}$ ne le présente plus comme une contrainte extérieure qui doit absolument être prise en compte. Ainsi, dans le rapport de 2012, le thème est évoqué mais il n'est plus central. Les réductions de $\mathrm{CO}_{2}$ consenties ne sont plus présentées comme des contraintes absolues, même si les efforts en ce sens sont démontrés. Par ailleurs, on peut noter le choix de couverture: une photographie d'un avion dans un aéroport. Lorsqu'on sait que le transport aérien est particulièrement émetteur de $\mathrm{CO}_{2}$, cette image ne peut être vue comme neutre et suggère un changement de positionnement de l'organisation vis-à-vis du changement climatique.

Le deuxième phénomène présenté par HSBC comme cause d'une réduction nécessaire de son impact environnemental est la crise financière de 2008. Il semblerait que cette crise ait secoué certains principes considérés comme des fondamentaux pour HSBC et, qu'en cette période, la banque ait été plus ouverte à des idées considérées jusqu'alors comme trop alternatives. Selon le rapport de 2008, cette crise a permis de mettre en avant l'importance des questions environnementales: "The importance of running a sustainable business for the long term has been highlighted by the global financial crisis.» $(2008$, p. 4) D'autres publications de l'organisation permettent de le confirmer, notamment la publication d'un rapport intitulé Building a Green Recovery (HSBC, 2009c) où il est expliqué que des idées écologistes qui semblaient bien loin des préoccupations d'une banque ne peuvent être négligées dans la conjoncture actuelle :

Over the past six months, the deepening global economic downturn has propelled ideas that were once on the margins of economic policy into the heart of decision-making: bank nationalisation, quantitative easing, and the focus of this report, low-carbon recovery. In July 2008, a group of far-sighted pioneers in the UK proposed a "Green New Deal" as a way of reviving demand, creating jobs and accelerating the transition to an economy consistent with the need to dramatically reduce greenhouse gas (GHGs) emissions over the coming decades. (HSBC, 2009c, p. 7) 

desquels on peut mesurer la consommation des ressources et la production de pollution. Différents éléments, comme la consommation de papier, d'énergie, d'eau et de pétrole, ainsi que le volume de déchets produits et d'émissions de carbones généré, peuvent alors être considérés. Étant donné que le thème environnemental sur lequel HSBC choisit de communiquer le plus régulièrement pendant la période étudiée est le changement climatique, nous allons nous concentrer sur les émissions de $\mathrm{CO}_{2}$. Des chiffres les concernant sont disponibles à partir du rapport de 2006 (HSBC, 2006, 2012).

Figure 3. - Nombres de tonnes de $\mathrm{CO}_{2}$ émises par HSBC de 2006 à 2012.

\begin{tabular}{|l|l|l|l|l|l|l|l|}
\hline Année & 2006 & 2007 & 2008 & 2009 & 2010 & 2011 & 2012 \\
\hline $\begin{array}{l}\text { Nombre de tonnes de } \mathrm{CO}_{2} \\
\text { émises }\end{array}$ & 813000 & 897000 & $954000^{2}$ & 991000 & 1017000 & 991000 & 963000 \\
\hline
\end{tabular}

Ces chiffres montrent que les efforts prônés pour réduire les émissions n'ont pas porté leurs fruits immédiatement. La réduction n'a été visible qu'à partir de 2011. À cette dernière s'ajoute un ambitieux objectif de baisse affiché dans le rapport de 2011. Il s'agit de réduire les émissions de carbone annuelles par employé d'une tonne en neuf ans afin d'afficher des taux bien plus écologiques en 2020. Mais si l'organisation mentionne ces efforts de réduction, notamment dans les tableaux récapitulatifs de données et dans les listes d'objectifs pour l'année à venir, dans le corps du texte décrivant les stratégies d'HSBC, il est notable que l'utilisation de lexèmes qui font directement référence à la réduction est limitée.

Nous avons ainsi relevé dans le corpus plusieurs exemples où des initiatives de réduction sont mentionnées, mais avec des formulations peu explicites. On trouve dans le rapport 
de 2003, parmi une liste d'objectifs que l'organisation s'est fixés, l'expression suivante : « to increase proportion of waste recycled» (2003, p. 18). Il s'agit d'efforts pour réduire les déchets de l'organisation, mais la formulation choisie permet d'utiliser le verbe « increase ", dont les connotations sont plus positives, plutôt que "reduce». On peut également trouver une expression qui concerne l'utilisation de papier : «In 2007, the use of paperless orders issued increased» (2007, p. 19). Ici encore le verbe "increase » est préféré. La négation porte sur l'adjectif « paperless ». La réduction est mentionnée de façon indirecte. Enfin, relevons cette expression du rapport de 2012 concernant les émissions de carbone d'HSBC : «Being carbon neutral means our operations contribute net zero carbon dioxide in the atmosphere » (2012). N'aurait-il pas été plus clair et plus logique dans ce cas d'utiliser l'expression «do not contribute to carbon dioxide in the atmosphere»? Mais HSBC est ainsi encore montrée comme sujet d'un verbe affirmatif et se présente comme contribuant à un mouvement positif plutôt que comme ne contribuant pas à quelque chose. Il semblerait que les expressions qui montrent HSBC comme sujet d'un verbe à la forme négative soient peu courantes dans les rapports considérés. Peut-être sont-elles évitées car elles pourraient suggérer que l'organisation n'est pas active. HSBC devrait au contraire être vue comme contribuant à un mouvement. Les verbes d'action seraient donc préférés, même pour indiquer des réductions de consommation. Afin de vérifier cette hypothèse à partir d'une étude empirique sur l'ensemble du corpus, il convient de se concentrer sur deux termes dont nous pouvons considérer l'utilisation de manière extensive.

\subsection{L'impact environnemental, un élément à réduire ?}

31 Le terme d'«environmental impact» est utilisé régulièrement dans les rapports sur la responsabilité sociale. On peut dire que le but de ces rapports est de présenter l'impact qu'une organisation a sur son environnement physique et parfois social. Ainsi, dans les instructions du Global Reporting Initiative figure la définition suivante: "'Sustainability reporting' is a broad term considered synonymous with others used to describe reporting on economic, environmental, and social impacts. " (Global Reporting Initiative, 2006, p. 3) On peut imaginer que le but d'HSBC, qui ne souhaite pas apparaître comme une entreprise polluante, est de réduire son impact environnemental. Pourtant, l'idée de réduction de l'impact environnemental n'est souvent pas mentionnée de manière explicite. Grâce au concordancier Antconc, nous avons relevé l'ensemble des expressions qui incluent le terme « environmental impact » dans notre corpus de rapports sur la responsabilité sociale.

Figure 4. - Expressions incluant le terme « environmental impact » dans les rapports HSBC.

\begin{tabular}{|l|l|}
\hline $2003 \mathrm{a}$ & $\begin{array}{l}\text { industry sectors with the potential for major, adverse social, ethical or environmental } \\
\text { impact }\end{array}$ \\
\hline $2003 \mathrm{~b}$ & conducting detailed environmental impact assessments \\
\hline $2003 \mathrm{c}$ & one of the leading companies in environmental impact management \\
\hline $2003 \mathrm{~d}$ & gathering data on our environmental impacts \\
\hline $2003 \mathrm{e}$ & reducing our environmental impacts \\
\hline
\end{tabular}




\begin{tabular}{|c|c|}
\hline $2003 \mathrm{f}$ & raise employee's awareness of their environmental impacts \\
\hline $2003 g$ & require environmental impact assessments \\
\hline $2003 \mathrm{~h}$ & minimising environmental impacts \\
\hline $2003 \mathrm{i}$ & management of its own direct and indirect environmental impacts \\
\hline $2003 \mathrm{j}$ & engaging $[. .$.$] on the economic, social and environmental impacts of these operations$ \\
\hline $2005 a$ & to manage the direct environmental impacts of our property portfolio \\
\hline $2005 b$ & influences the environmental impacts of our suppliers \\
\hline $2005 \mathrm{c}$ & environmental impact data, which has been verified separately \\
\hline $2007 \mathrm{a}$ & information relevant to $\mathrm{HSBC}$ 's direct environmental impact \\
\hline $2007 b$ & examine our own environmental impacts \\
\hline $2007 \mathrm{c}$ & to reduce HSBC's direct environmental impact \\
\hline $2007 d$ & concerns $[. .$.$] over environmental impacts$ \\
\hline 2007e & the policy defines areas of high environmental impact \\
\hline $2008 \mathrm{a}$ & managing the social and environmental impact of our business \\
\hline $2008 b$ & mitigating any potential social or environmental impacts \\
\hline $2008 \mathrm{c}$ & to reduce the direct environmental impacts \\
\hline $2008 \mathrm{~d}$ & energy use is our most significant environmental impact \\
\hline $2008 \mathrm{e}$ & to reduce environmental impact \\
\hline $2008 \mathrm{f}$ & to deliver cost-effective environmental impact reduction programmes \\
\hline $2008 \mathrm{~g}$ & the management of our direct environmental impacts \\
\hline $2010 \mathrm{a}$ & measure and report on our group-wide environmental impact \\
\hline $2010 b$ & to reduce environmental impact of our business travel \\
\hline $2012 \mathrm{a}$ & to report their environmental impacts \\
\hline
\end{tabular}

32 Nous faisons apparaître les prédicats en gras afin de s'interroger sur la catégorie qui est la plus utilisée avec le terme "environmental impact». Les prédicats signifiant la réduction ne sont pas absents de ce tableau: "reduce» est relevé sous forme verbale cinq fois (2003e, 2007c, 2008c, 2008e, 2010b) et sous forme nominale une fois (2008f). Deux autres verbes synonymes sont utilisés : "to minimise» (2003h) et «to mitigate» (2008b). Cependant d'autres types de prédicats sont souvent préférés. Dans la majorité des cas, ces 
prédicats ont une signification liée au contrôle de l'impact environnemental plutôt qu'à sa réduction : « to manage » (2003c, 2003i, 2005a, 2008a, 2008g), « to assess » (2003b, 2003g), «to gather data on » (2003d), «to verify» (2005c), "to examine» (2007b), «to measure " (2010a), « to report » (2010a, 2012a). Le champ sémantique du contrôle semble primer. Les prédicats restants sont liés à cette volonté de contrôle de l'impact puisqu'ils signifient la volonté de mettre en place des mesures le permettant: " raise employee's awareness of " (2003f), «engaging on " (2003j), «influence " (2005b), "define areas of » (2007e). Mais le terme qui est préféré de loin à celui de "environmental impact » pour se référer aux mesures écologiques est, comme mentionné auparavant, celui de "sustainability». Ce pourrait ne pas être sans rapport avec le fait de vouloir présenter HSBC dans une position valorisante et active par rapport aux questions écologiques.

\subsection{La valeur du terme " sustainability »}

Différentes hypothèses pourraient expliquer le succès de ce terme, mais le fait qu'il puisse être utilisé dans des phrases affirmatives, qui montrent HSBC en position active, ne nous y semble pas étranger. Avec ce terme, toute mention de réduction peut être occultée. " Sustainability ", bien que mal défini, est très apprécié par l'organisation qui peut ainsi exprimer le fait d'être moins polluant et d'utiliser moins de ressources avec un terme qui suggère quelque chose de positif, un mouvement à encourager, à développer et à augmenter. On peut y voir une possible explication au succès du concept.

En reprenant le tableau 1, l'on s'aperçoit que les occurrences du terme sont particulièrement nombreuses en 2007. On peut donc se concentrer sur celles-ci pour tenter de dégager des tendances concernant le type d'expressions dans lesquelles le terme est utilisé. Après avoir relevé l'ensemble des expressions contenant le terme " sustainability» dans le rapport de 2007, nous avons classé ces dernières par catégories sémantiques.

Figure 5. - Expressions contenant le terme « sustainability » référant aux actions de l'entreprise dans le domaine.

\begin{tabular}{|l|l|l|}
\hline \multicolumn{2}{|l|}{ Action } & 1 \\
\hline \multirow{4}{*}{ Organisation } & sustainability teams & 4 \\
\cline { 2 - 3 } & sustainability reporting & 2 \\
\cline { 2 - 3 } & sustainability reports & 1 \\
\cline { 2 - 3 } & sustainability expenditure & 1 \\
\cline { 2 - 3 } & verify sustainability & 1 \\
\cline { 2 - 3 } & record on sustainability & 1 \\
\cline { 2 - 3 } & proof of sustainability & 1 \\
\cline { 2 - 3 } & long-term sustainability of the organisation & \\
\hline
\end{tabular}




\begin{tabular}{|c|c|c|}
\hline \multirow{12}{*}{ Intégration } & $\begin{array}{l}\text { we want sustainability to be ingrained in our corporate } \\
\text { strategy }\end{array}$ & 1 \\
\hline & made sustainability a key component of our strategy & 1 \\
\hline & include a sustainability aspect & 1 \\
\hline & embed(ding) sustainability into its/our business & 4 \\
\hline & include sustainability among these values & 1 \\
\hline & commitment to sustainability & 1 \\
\hline & integrate sustainability & 1 \\
\hline & integration of sustainability & 1 \\
\hline & engagement on sustainability & 2 \\
\hline & embed sustainability & 1 \\
\hline & integrate sustainability issues & 1 \\
\hline & sustainability is essential to recruiting and retaining & 1 \\
\hline \multirow{2}{*}{ Non-intégration } & sustainability cannot be embedded & 1 \\
\hline & pay lip service to sustainability & 1 \\
\hline \multirow{5}{*}{ Définitions des règles } & HSBC's sustainability policies & 1 \\
\hline & HSBC's sustainability risk policies & 1 \\
\hline & sustainability risk sector policies & 3 \\
\hline & sustainability policies & 3 \\
\hline & corporate policies relating to sustainability & 1 \\
\hline \multirow{7}{*}{$\begin{array}{l}\text { Définitions des stratégies et } \\
\text { buts }\end{array}$} & environmental sustainability initiatives & 1 \\
\hline & sustainability efforts & 1 \\
\hline & sustainability strategies & 1 \\
\hline & sustainability strategy & 6 \\
\hline & sustainability-related targets & 1 \\
\hline & sustainability solutions & 2 \\
\hline & sustainability performance & 2 \\
\hline
\end{tabular}




\begin{tabular}{|c|c|c|}
\hline & opportunities linked to sustainability & 1 \\
\hline & address sustainability challenges & 1 \\
\hline \multirow{17}{*}{ Gestion des risques et impacts } & how we manage sustainability risk & 1 \\
\hline & managing sustainability risk & 1 \\
\hline & management of sustainability risk & 1 \\
\hline & improved sustainability risk management & 1 \\
\hline & sustainability risk management & 1 \\
\hline & sustainability Risk Managers & 2 \\
\hline & sustainability risk ratings & 2 \\
\hline & sustainability risk rating & 1 \\
\hline & identify and assess sustainability risks & 2 \\
\hline & management of [...] sustainability risk & 1 \\
\hline & how $\mathrm{HSBC}$ views sustainability risk & 1 \\
\hline & a section on sustainability risk & 1 \\
\hline & where there is high sustainability risk & 1 \\
\hline & focuses on sustainability risk & 1 \\
\hline & the sustainability impacts were unacceptable & 1 \\
\hline & $\begin{array}{l}\text { palm oil can be associated with a variety of sustainability } \\
\text { impacts }\end{array}$ & 1 \\
\hline & track indirect sustainability impacts & 1 \\
\hline \multirow{4}{*}{ Amélioration } & supports the sustainability & 1 \\
\hline & promoting sustainability & 1 \\
\hline & building sustainability & 1 \\
\hline & engage with $\mathrm{HSBC}$ on the subject of sustainability & 1 \\
\hline \multirow{3}{*}{$\begin{array}{l}\text { Occupation de la première } \\
\text { position }\end{array}$} & leading brands in sustainability & 1 \\
\hline & leading brands in corporate sustainability & 1 \\
\hline & leading brands for sustainability & 1 \\
\hline
\end{tabular}


Figure 6. - Expressions contenant le terme « sustainability » se référant au savoir développé dans le domaine.

\begin{tabular}{|c|c|c|}
\hline \multicolumn{3}{|c|}{ Savoir } \\
\hline \multirow{5}{*}{$\begin{array}{l}\text { Construction } \mathrm{du} \\
\text { savoir }\end{array}$} & embed consistent sustainability learning & 1 \\
\hline & knowledge of sustainability & 1 \\
\hline & summary of sustainability training & 1 \\
\hline & trained on sustainability & 1 \\
\hline & provision of sustainability context & 1 \\
\hline \multirow{6}{*}{ Définition } & What sustainability means to $\mathrm{HSBC}$ & 1 \\
\hline & sustainability is about making decisions & 1 \\
\hline & HSBC's vision and mission on sustainability & 1 \\
\hline & appreciation of sustainability & 1 \\
\hline & $\begin{array}{l}\text { we refer to «corporate sustainability" rather than «corporate } \\
\text { responsibility " as it describes more succintly ... }\end{array}$ & 1 \\
\hline & finance/lending strategy is the most critical sustainability issue & 1 \\
\hline \multirow{4}{*}{$\begin{array}{l}\text { Thème } \\
\text { discussion }\end{array}$} & dialogue on sustainability issues & 1 \\
\hline & sustainability topics & 1 \\
\hline & sustainability issues & 1 \\
\hline & reference to sustainability & 1 \\
\hline
\end{tabular}

Ces deux tableaux permettent de faire apparaître deux grandes catégories d'usage du terme "sustainability»: ceux qui présentent les actions de l'organisation et ceux qui mentionnent le développement du savoir dans le domaine. Les éléments d'organisation à l'intérieur d'HSBC sont mentionnés avec une référence aux équipes spécialisées, aux rapports publiés, aux procédures de vérifications et autres documents ou unités spécialisées. Puis on trouve de nombreuses expressions visant à signifier que ce thème doit être au cœur de la stratégie d'HSBC et influencer profondément toutes ses actions plutôt que d'être considéré comme annexe («we want sustainability to be ingrained in our corporate ethos»; "embedding sustainability into its business»). Par opposition, deux expressions ("sustainability cannot be embedded "; "to pay lip service to sustainability ») permettent de faire référence à ce qu'HSBC veut éviter : une intégration superficielle de 
ces problématiques. La mise en place de règles, la définition de stratégies et d'objectifs montrent la volonté d'HSBC d'agir sur le sujet, tout comme la référence à la gestion des risques et impacts liés. Le fait qu'HSBC s'inscrit dans une dynamique positive dans ce cadre est signifié au moyen d'expressions concernant les processus d'amélioration et l'occupation d'une position privilégiée de la banque par rapport à ses concurrents. Dans le domaine du savoir, on trouve des expressions sur sa construction, notamment avec la mention de formations, des définitions du terme ainsi que quelques expressions qui mentionnent « sustainability » comme un thème de discussion.

L'organisation est ainsi montrée comme active, mettant en place des équipes responsables, définissant des objectifs, évaluant, développant le savoir sur le sujet. Il ne s'agit pas simplement de se montrer comme consommant moins mais d'insister sur le fait qu'il s'agit d'un risque maîtrisé. La banque apparaît ainsi dans un rôle d'entité gestionnaire plutôt que comme une organisation qui agit moins pour consommer moins. Une idée de performance se dégage de la plupart de ces expressions. L'entreprise peut se montrer comme active et bien placée dans le domaine plutôt que d'expliquer concrètement qu'elle pollue de moins en moins ou utilise de moins en moins de ressources.

\subsection{Compétition pour la protection de l'environnement}

Comme nous avons pu le voir avec l'exemple de "sustainability », HSBC a tendance à se comparer à d'autres institutions équivalentes, afin de montrer que par rapport à elles, elle est performante sur ce sujet, comme sur d'autres sujets. Le lexème de "leader » est alors utilisé régulièrement. HSBC participe ainsi à de nombreux concours et cite régulièrement les prix reçus, afin de démontrer la qualité de ses performances dans le domaine: " HSBC was named one of the top five global companies for accountability in annual ratings conducted by AccountAbility and CSR Network. HSBC was lauded for climate change strategy, with a score of 67 per cent compared with 38 per cent in the financial sector.» (2007, Content page)

Les stratégies permettant de réduire l'impact environnemental d'HSBC semblent particulièrement appréciées dans le milieu si l'on en juge d'après les nombreux prix cités dans les rapports. Il s'agit d'un thème qui a été considéré prioritaire entre 2006 et 2010 et les investissements consentis par l'organisation semblent avoir porté leurs fruits d'après les extraits qui présentent des listes de récompenses attribuées à la banque ${ }^{3}$. Afin de favoriser l'esprit de compétition parmi les employés sur ces thèmes, l'organisation choisit aussi d'organiser des concours pour récompenser un projet qui répond bien aux objectifs de la stratégie "sustainability $»^{4}$. Il s'agit donc de transformer une contrainte en opportunité en utilisant ce thème comme moteur de compétition.

HSBC propose une autre façon de voir les contraintes liées au changement climatique comme moteur de compétition. Cette nouvelle perspective sur la question est déjà mentionnée dans les rapports précédents, mais à partir du rapport sur la responsabilité sociale de 2010, on peut trouver un chapitre intitulé "Climate Business » et cet aspect constitue une partie importante des rapports. Il s'agit de développer l'activité économique grâce au changement climatique comme expliqué dans l'extrait suivant :

We define climate business as the goods and services that will thrive in the transition to a

low carbon economy. It comprises four major areas of opportunity: 
- increasing the share of low carbon energy production, such as bio-energy, solar and wind;

- improving energy efficiency in buildings, industry and transport, as well as energy storage;

- adapting to the impacts of climate change, particularly in agriculture, infrastructure and water; and

- providing climate finance, including environmental markets, debt and equity investment and insurance. (2011, p. 15)

Bien loin d'associer de moindres émissions de carbones à une moindre activité économique, la croissance rendue possible grâce au changement climatique est soulignée. De façon quelque peu paradoxale, dans le discours de la banque, il est devenu un argument économique, un moteur de la croissance.

\section{Conclusion}

41 Malgré le développement important de la communication sur l'environnement et sa protection dans les discours de grandes banques internationales comme HSBC, l'idée qu'il est possible de faire plus avec moins de moyens matériels et moins de croissance économique reste transgressive. Si une réduction de l'utilisation de ressources naturelles peut être justifiée sans mettre en péril la cohérence du discours d'HSBC, grâce à des arguments qui ont souvent peu de liens avec la protection de l'environnement en soi, HSBC reste, avant tout, dans une optique de croissance économique et de favorisation de la consommation. Cela a un impact sur les formulations choisies pour décrire la moindre consommation de ressources. La réduction ne doit pas être exprimée frontalement mais plutôt par des moyens détournés. Si la communication des entreprises sur les thématiques environnementales semble suggérer qu'elles sont prêtes à s'engager pour la protection de l'environnement, elles peuvent difficilement accepter les principes impliquant une remise en question du cœur même de leur activité. L'idée, fondamentale pour ceux dont la priorité est de protéger l'environnement, qu'il faut consommer moins de ressources et se méfier de l'impératif de croissance économique, reste assez étrangère à leurs discours. L'exemple du traitement réservé au thème du changement climatique dans le discours externe de la banque est particulièrement parlant de ce point de vue. $\mathrm{Si}$, dans un premier temps, HSBC insiste simplement sur les efforts consentis pour réduire ses taux d'émissions de $\mathrm{CO}_{2}$ en vue de limiter ce phénomène dont la menace semble réelle, depuis 2010, c'est dans une optique entrepreneuriale que le thème est considéré. La réduction des émissions est génératrice d'activité économique et c'est de ce point de vue que la question est présentée, dans un long chapitre intitulé «Climate Business ». Cela peut paraitre paradoxal. On pourrait répondre à $\mathrm{HSBC}$ avec les mots de Schumacher et mesurer ainsi la faille qui continue à séparer ceux qui se préoccupent avant tout de la survie du système écologique et ceux qui se soucient avant tout de la préservation du système économique : "If a high-growth economy is needed to fight the battle against pollution which itself appears to be the result of high-growth, what hope is there of ever breaking out of this extraordinary circle? » (Schumacher, 1972) 


\section{BIBLIOGRAPHIE}

\section{Sources primaires}

HSBC, <www.hsbc.co.uk> (consulté le 4 mai 2013).

HSBC, HSBC in Society - Corporate Social Responsibility Report 2003, Londres, HSBC Holdings Plc., 2003.

HSBC, HSBC Corporate Social Responsibility Report 2004, Londres, HSBC Holdings Plc., 2004.

HSBC, HSBC Corporate Social Responsibility Report 2005, Londres, HSBC Holdings Plc., 2005.

HSBC, HSBC Corporate Responsibility Report 2006, Londres, HSBC Holdings Plc., 2006.

HSBC, HSBC Sustainability Report 2007, Londres, HSBC Holdings Plc., 2007.

HSBC, HSBC and Carbon Neutrality, Londres, HSBC Holdings plc., 2008a.

HSBC, HSBC Holdings plc. Sustainability Report 2008, Londres, HSBC Holdings Plc., 2008b.

HSBC, HSBC Holdings plc. Sustainability Report 2009, Londres, HSBC Holdings Plc., 2009a.

HSBC, A Climate for Recovery - The Colour of Stimumus Goes Green, Londres, HSBC Bank plc., $2009 \mathrm{~b}$.

HSBC, Building a Green Recovery - Governments Allocate USD470bn - and Counting ..., Londres, HSBC

Holdings plc., 2009c.

HSBC, HSBC Holdings plc. Sustainability Report 2010, Londres, HSBC Holdings Plc., 2010.

HSBC, HSBC Holdings plc. Sustainability Report 2011, Londres, HSBC Holdings Plc., 2011a.

HSBC, Climate Partnership Review 2010, Londres, HSBC Holdings plc., 2011b.

HSBC, HSBC Holdings plc. Sustainability Report 2012, Londres, HSBC Holdings Plc., 2012.

\section{Sources secondaires}

BIROS Camille, Contribution à l'étude du discours environnemental : les organisations et leurs discours au Royaume-Uni, thèse d'études anglaises, Bordeaux 2, 2011.

CARSON Rachel, Silent Spring, New York, Houghton Mifflin, 2002 [1962].

CARTER Neil, « Greening the Mainstream: Party Politics and the Environment », Environmental Politics, vol. 22, $\mathrm{n}^{\circ}$ 1, 2013, p. 73-94.

Charaudeau Patrick \& maingueneau Dominique, Dictionnaire d'analyse de discours, Paris, Seuil, 2002.

EMERSON Ralph Waldo, Nature, Boston et Cambridge, James Munroe and Company, 1849.

GLOBAL REPORTING INITIATIVE, G3 Sustainability Reporting Guidelines, Amsterdam, Global Reporting Initiative, 2006.

IPCC [solomon et al. (éds)], Climate Change 2007: The Physical Science Basis. Contribution of Working Group I to the Fourth Assessment Report of the Intergovernmental Panel on Climate Change, Cambridge, CUP, 2007.

KRIEG-PLANQUE Alice, « La formule développement durable : un opérateur de neutralisation de la conflictualité », Langage et Société, $\mathrm{n}^{\circ} 134,2010$, p. 5-29. 
LATOUCHE Serge, Survivre au développement : de la décolonisation de l'imaginaire économique à la construction d'une société alternative, Paris, Mille et une nuits, 2004.

LEAL FILHO Walter, Communicating Sustainability, Francfort, Peter Lang CombH, 2000.

LEOPOLD Aldo, A Sand and County Almanac with Essays on Conservation from Round River, Oxford, OUP, 1966 [1949].

MAINGUENEAU Dominique \& cossutA Frédéric, « L'analyse des discours constituants », Langages, $\mathrm{n}^{\circ}$ 117, 1995, p. 112-125.

MARSH George Perkins, Man and Nature, or, Physical Geography as Modified by Human Action, New York, Charles Scribner \& Co., 1867.

MEADows Donella H. et al., The Limits to Growth, New York, Universe Books, 1972.

PARK Chris, Oxford Dictionary of Environment and Conservation, Oxford, OUP, 2008.

PINCHот George, Breaking New Grounds, New York, Harcourt, Brace and Co., 1947.

RESCHE Catherine, «L'implicite dans le discours des grandes entreprises sur leur responsabilité sociale ", Les Cahiers de l'ILCEA, nº 9, 2007, p. 7-47.

SCHUMACHER Ernst Friedrich, Small Is Beautiful, Kindle Version, 1972.

STERN Nicholas, The Economics of Climate Change: The Stern Review, Cambridge, CUP, 2007.

THOREAU Henry David, Walden, Princeton, Princeton U. P., 1971 [1854].

TOURNIER Jean, Précis de lexicologie anglaise, Paris, Ellipses, 2004.

WORLD COMMISSION ON ENVIRONMENT AND DEVELOPMENT, Our Common Future / The World Commission on Environment and Development, Oxford et New York, OUP, 1991.

\section{NOTES}

1. "we formalised our commitment to sustainability in 1992 by becoming a founder signatory to the UN Environment Programme Statement by Financial Institutions on the Environment and Sustainable Development » $(2003$, p. 3$)$; « the promotion of good environmental practice and sustainable development » (2003, p. 9) ; « founder signatory to the United Nations Environment Programme Statement by Financial Institutions on the Environment and Sustainable Development » $(2003$, p. 11$)$; «HSBC is committed to playing its part in making in sustainable development a reality-meeting the needs of the present without compromising those of the future" (2003, p. 13) ; "In order to fulfill our commitments to sustainable development, we have to understand the effects of our working practices» $(2003$, p. 14) ; «In 2003 we also created a number of opportunities for institutional investors to meet and question companies on their progress and plans in respect of sustainable development » $(2003$, p. 21$)$; « Awareness and appreciation of environmental issues are vital to the sustainable development of both our business and the communities in which we operate» (2003, p. 40).

2. Il est précisé dans le rapport que le mode de calcul ayant changé, le chiffre de 2007 n'est plus valable et devrait être de 953000.

3. «In 2008, HSBC's North America headquarters building in Mettawa Illinois, received the Leadership in Energy and Environmental Design (LEED) gold certification from the US Green Building Council » (2008, p. 23); «In 2009, HSBC was listed number one amongst financial institutions for its climate change strategy and reporting of emissions in the Carbon Disclosure Project; we were again listed in the FTSE4Good and Dow Jones sustainability indices » $(2009$, p. 2$)$; «Our Climate Change team was ranked number one 
for the third year in a row by Thomson Reuters EXTEL awards. Nick Robins, Head of Climate Change Research, was also voted the number one climate change analyst for the third year running, out of 42 analysts " (2012, p. 14).

4. «In 2012, we launched a new operational sustainability award to celebrate 'Sustainability Excellence' This recognises a project that 'strengthens HSBC's role as a responsible institution and delivers value to the communities, customers and shareholders'. » (2012, p. 21)

\section{RÉSUMÉS}

Les textes fondateurs du mouvement écologique soulignent l'idée qu'il faut tenter de réduire la consommation des ressources afin de préserver les équilibres naturels. Dans un système économique fondé sur l'idée de croissance et de hausse constante de la consommation, cette idée constitue, en soi, une transgression. Allant à l'encontre de valeurs économiques fondamentales, elle pourrait perturber l'ordre établi et a donc rarement été soutenue par les grands acteurs du système que sont les banques, et en particulier une grande banque internationale comme HSBC. Pourtant, crises économiques, énergétiques et écologiques contribuent à ébranler les normes dans le domaine. Une étude des textes de communication externe de la banque HSBC permet de constater qu'elle intègre progressivement des principes liés à la réduction de la consommation, phénomène dont nous étudions les manifestations. Nous commençons par présenter succinctement les origines de cette idée en évoquant quelques textes qui ont beaucoup marqué le mouvement environnemental parmi lesquels deux se distinguent particulièrement: Walden de Thoreau et Small is Beautiful de Schumacher. Dans un deuxième temps nous proposons une analyse textuelle de rapports de communication externe d'HSBC et présentons les critères mis en avant par cette banque pour justifier une baisse dans la consommation des ressources. Mettre à jour certaines ambiguïtés dans le discours de la banque permet néanmoins de faire apparaître que l'idée qu'il est possible de faire plus avec moins de moyens matériels reste transgressive.

Founding texts of the ecological movement highlight the idea that reducing the consumption of resources is necessary to the preservation of a healthy environment. In an economic system based on the idea of growth and every-increasing consumption, such thinking can only be transgressive in that it goes against fundamental economic values and threatens to disrupt the established order. As such, it has rarely been defended by the banking sector, which has important stakes in preserving the system, and even less so by a big international bank like HSBC. However, the emergence of different types of crisis (economic, energetic and ecological) has contributed to weakening established ideas in the domain and an analysis of the language and discourse of HSBC's external communication reveals a gradual integration of principles linked to the reduction of consumption. We begin by briefly presenting the origins of such thinking by referring to certain founding texts associated with the environmental movement, notably Walden by Thoreau and Small is beautiful by Schumacher. We then go on to propose a textual analysis of HSBC's external communication reports and present the criteria put forward by the bank to justify a decrease in the consumption of resources. Shedding light on some ambiguities in the bank's discourse nevertheless shows that advocating the possibility of doing more with less material means continues to remain a form of transgression. 
INDEX

Mots-clés : transgression, communication environnementale, écologie, HSBC, consommation, croissance

Keywords : transgression, environmental communication, ecology, HSBC, consumption, growth

\section{AUTEUR}

\section{CAMILLE BIROS}

Agrégée d'anglais, Camille Biros est maître de conférences au département des langues de la faculté de médecine et de pharmacie de l'université Joseph Fourier (Grenoble 1) où elle donne des cours d'anglais de la médecine, de la pharmacie et des biotechnologies. Membre du laboratoire Institut des langues et des cultures d'Europe et d'Amérique (Grenoble 3), ses recherches portent sur les discours spécialisés du domaine de l'environnement. 